\title{
Ultrafast large-scale chemical sensing with CMOS ISFETs: a level-crossing time-domain approach
}

\author{
Yan Liu, Member, IEEE, Timothy G. Constandinou, Senior Member, IEEE and \\ Pantelis Georgiou, Senior Member, IEEE
}

\begin{abstract}
The introduction of large-scale chemical sensing systems in CMOS which integrate millions of ISFET sensors have allowed applications such as DNA sequencing and fine-pixel chemical imaging systems to be realised. Using CMOS ISFETs provides advantages of digitisation directly at the sensor as well as correcting for non-linearity in its response. However, for this to be beneficial and scale, the readout circuits need to have the minimum possible footprint and power consumption. Within this context, this paper analyses an ISFET based pH-to-time readout using an inverter in the time-domain as a level-crossing detector and presents a $32 \times 32$ array with in-pixel digitisation for $\mathrm{pH}$ sensing. The inverter-based sensing pixel, controlled by a triangular waveform, converts the $\mathrm{pH}$ response into a timedomain signal whilst also compensating for sensor offset and thus resulting in an increase in dynamic range. The sensor pixels interface to a 15-bit asynchronous column-wise time-todigital converter (TDC), enabling fast asynchronous conversion whilst using minimal silicon area. Parallel outputs of 32 TDC interfaces are serialised to achieve fast data throughput. This system is implemented in a standard $0.18 \mu \mathrm{m}$ CMOS technology, with a pixel size of $26 \mu \mathrm{m} \times 26 \mu \mathrm{m}$ and a TDC area of $26 \mu \mathrm{m} \times$ $180 \mu \mathrm{m}$. Additionally, we investigate the use of additional offset compensation by having half of the array implemented with the floating gate tied down via a well diode. Measured results demonstrate the system is able to sense reliably with an average pH sensitivity of $30 \mathrm{mV} / \mathrm{pH}$, whilst being able to compensate for sensor offset by up to $\pm 7 \mathrm{~V}$. A resolution of $0.013 \mathrm{pH}$ is achieved and noise measurements show an integrated noise of $0.08 \mathrm{pH}$ within $2-500 \mathrm{~Hz}$ and SFDR of $42.6 \mathrm{~dB}$. The total power consumption of the system is measured to be $11.286 \mathrm{~mW}$ when operating at a high frame rate of 1 KFPS.
\end{abstract}

\section{INTRODUCTION}

W ITHIN the past decade, we have witnessed microelectronic and micro-system technologies having a significant impact on biomedical applications such as electrochemical Lab-on-Chip systems, neural interfaces and DNA sequencing platforms [1]-[3]. In addition to this, we are now seeing the implementation of systems which include large-scale arrays of miniature sensors or electrodes which are also integrated with low noise analogue front-ends capable of direct conversion and subsequent processing. The realisation of such systems in commercially available CMOS technology has allowed reliable and repeatable fabrication at low cost, in addition to the opportunity for large-scale integration and scaling which is seeing transistor feature sizes reduce according to Moore's

Y. Liu is with Department of Micro-Nano Electronics, Shanghai Jiaotong University, China, Y. Liu, T. G. Constandinou and P. Georgiou are with the Centre for Bio-Inspired Technology, Department of Electrical and Electronic Engineering, Imperial College London, South Kensington Campus, London SW7 2AZ, UK (e-mail:yan.liu06@imperial.ac.uk).

Manuscript received XX; revised YY.
Law. Furthermore, for lab-on-chip systems, this scaling has seen the miniaturisation of the sensor interface and therefore a reduction in the associated parasitics, improving sensitivity. This has enabled high speed parallel sensing and integration of instrumentation together with CMOS-based chemical sensors such as the Ion-Sensitive Field Effect Transistor (ISFET).

ISFETs are a class of CMOS-compatible potentiometric chemical sensors, which have been extensively studied and used commercially for DNA sequencing or SNPs (Single Nucleotide Polymorphisms) detection [2], [4]. Owing to the decreasing feature size of CMOS technology, millions of $\mu \mathrm{m}$ size ISFETs can now be integrated into a mm-size silicon chip, with fully on-chip interfacing and processing circuitry [2], [5]-[7]. High density integration of ISFET arrays in CMOS greatly reduces cost, but also introduces design challenges for scalability of the sensor interface [8].

Conventional analogue-based ISFET readouts have limitations in scaling due to the circuit complexity required to compensate non-idealities and speed as a result of parasitics [9]. In-pixel bias and sense circuitry therefore impose limits as to how small the pixel sizes can shrink. In optical imaging, Active Pixel Sensor (APS) based readouts simplify the inpixel structure, together with column or array-level Correlated Double Sampling (CDS) for reducing mismatch in the sensor output. This architecture has also been applied to chemical sensing arrays [6], [7], [10]. Here, the voltage on the floating gate is converted to a current, with a sampling capacitor being charged via a source-follower or common source configuration. However, this introduces speed limitations and non-linearity. Furthermore, compensation schemes for sensor offset are usually performed via resetting the floating gate voltage or by biasing the reference electrode. This poses a challenge for continuous monitoring applications that require fully-integrated biasing [6], [7], [11].

On the other hand, a completely time-based ISFET readout provides the possibility for a maximally-compact sensor readout, whilst also overcoming speed limitations. However, the Pulse Width Modulation (PWM) output that is typically employed in such readouts poses certain challenges. Special consideration is needed here for readout synchronization, parasitics due to a large array, and large sensor offsets need compensation [12].

In this paper, we extend our previous work in [14], providing detailed analysis on the offset compensation, noise performance, linearity of the inverter-based ISFET readout, and discuss design considerations for large-scale ISFET arrays. To demonstrate the proposed scheme, sensor pixels were designed 


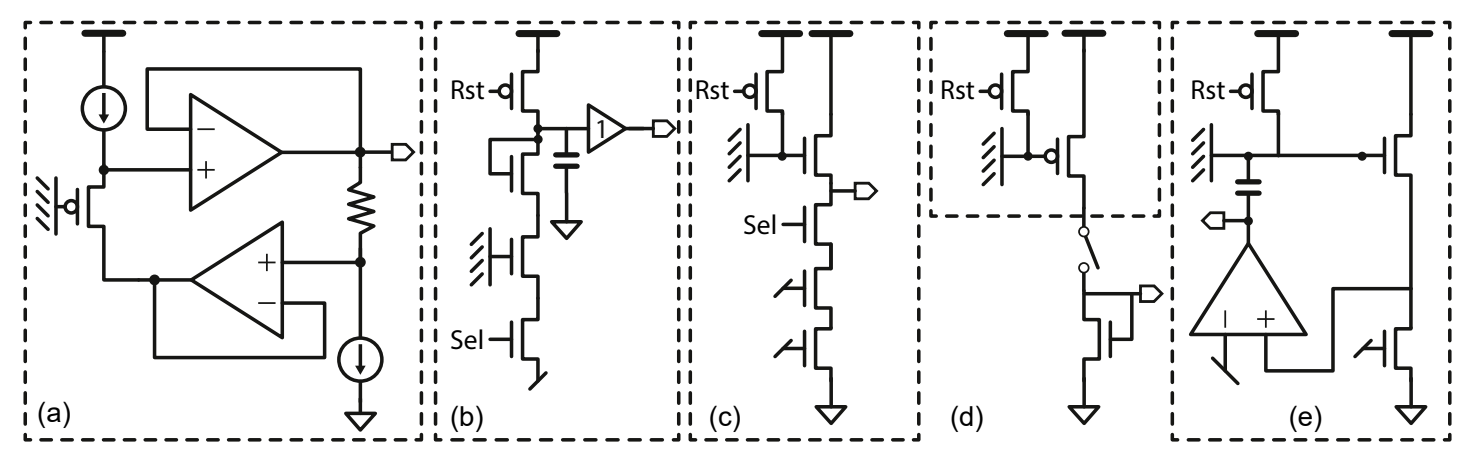

Fig. 1. Typical ISFETs readout circuits with dashed boxes indicating the in-pixel structure: (a) Source drain follower [13]; (b) time-based readout with current discharging output nodes [6]; (c) PWM-based interface with time output [12]; (d) in-pixel common-source configuration with external interface [10]; (e) Closed-loop readout with ISFETs within the loop [11].

to compensate $\pm 5 \mathrm{~V}$ sensor offset with a $0.01 \mathrm{pH}$ resolution. To convert the PWM output, an asynchronous 15-bit timeto-digital converter was proposed to provide fast and direct sensor output conversion. A $32 \times 32$ sensor array has been implemented with a simulated maximum sampling rate of $5 \mathrm{~K}$ frames per second. Measured results showed distribution of offset across the entire array has standard deviation of $0.6 \mathrm{~V}$ and maximum $7 \mathrm{~V}$ offset can be compensated by the proposed scheme. The measured pH sensitivity was 70 LSB(least significant bits) $/ \mathrm{pH}$, equivalent to $30 \mathrm{mV} / \mathrm{pH}$. The integrated noise indicated minimum resolution of $\mathrm{pH}$ of 0.08 is achieved with bandwidth of 2-500 Hz, and power consumption of $11.286 \mathrm{~mW}$ when operating at a high frame rate of 1 KFPS (1000 frames per second).

This paper is organised as follows: Section II provides an overview to readout circuits for ISFET sensors, and discusses design considerations in large-scale arrays; Section III describes the operation of a $\mathrm{pH}$-time converter with detailed analysis of offset compensation and noise; Section IV describes sensor realization, the time-to-digital converter and the system implementation; Section V provides both simulation and measured results and Section VI presents the final discussion and conclusion.

\section{OVERVIEW OF ISFET READOUT CIRCUITS}

ISFETs are potentiometric sensors, in which the sensed potentials directly relate to the ion-concentration [13] of the $\mathrm{pH}$ solutions they are measuring. The chemical induced voltage change $V_{\text {chem }}$ is related to the bulk $\mathrm{pH}$ value, given by:

$$
\frac{d V_{\text {chem }}}{d p H}=-2.3 \frac{k T}{q}\left(\frac{1}{1+\alpha-\beta}\right)
$$

where $\alpha$ is the coefficient for site binding model and $\beta$ is the reduction coefficient for counter-ion effect [13], [15].

Compared to non-CMOS based ISFETs or modified CMOS ISFETs, ISFETs in unmodified CMOS boast a lower cost, simplified integration [13], and the ability to implement additional floating gate nodes - this enables the sensor to be decoupled from the electronic biasing [8], and provides extra control path [11] for further compensation. However this comes at the cost of non-linear characteristics, such as offset and drift, due to the intrinsic passivation layer as sensing membrane and the floating gate structure [8]. Therefore various ISFET readout circuits have been proposed to accommodate or minimize nonideal effects whilst maintaining a low power budget in a largescale system [16].

The source drain follower circuits in Fig. 1[a] provide good linearity, by providing constant biasing and suppressing parasitic capacitance from source, drain and bulk nodes to the floating gate, thereby clamping the output voltage to the chemical input voltage [13]. However due to complexity and power consumption of the circuits, this has not been applicable to large-scale sensing array systems. The source follower readout [5], [7] in Fig. 1[c] outputs a direct chemical voltage change, however, requires extra amplification for data conversion, such that the noise in the following stages is directly referred to the chemical signal. The common-source based readout converts the chemical voltage to current output, then outputs a voltage by discharging the load capacitors [6] Fig. 1[b], or outputs a current via current conveyors [5], [17] Fig. 1[d]. These require a constant biasing during the readout and introduce non-linearity and signal reduction due to the capacitor ratio in the floating gate. Therefore, their noise performance is directly related to the biasing current, and power consumption. Moreover, such open-loop solutions do not achieve sufficient linearity, therefore $\mathrm{pH}$-to-voltage mapping and calibration are needed. Compared to conventional APS sensor pixels, the chemical sensors exhibited drift signal which overlap the signal spectrum [8], therefore the CDS (correlated double sampling) circuits used to remove offset and reset noise is not effective for drift cancellation, and offset removal for chemical sensors due to the mismatch induced could be larger than the rail supply [6].

ISFETs can also be part of the loop [11], [18]. Capacitive feedback with large open-loop gain can suppress the variation on the floating gate, and also provide gain to the signal with high linearity as shown in Fig. 1.e. These circuits still require offset compensation before establishing the feedback loop, and internal amplifiers used in the loop have power and speed limitations.

It is arguable that noise and linearity performance of readout circuits will be dominant in the sensing system, when chemical noise and drift are much higher than electronic noise [8]. Moreover, chemical systems already introduce large non- 
linearity due to double layer, site-bonding models, and crowd ion effect [15]. However, for large-scale systems with high sampling rate, the power consumption of the total sensing arrays can easily exceed $\mathrm{mW}$ [17], where a significant resource is required for real time non-linearity correction even when the $\mathrm{pH}$ change is small. With a scaled-down sensor size, electrical noise may dominate the noise contribution and affect the linearity due to increased flicker noise and large gate capacitance. Therefore minimizing non-linearity and noise without a high power budget is preferable for ultra-high speed chemical sensing.

On the other hand, offsets across individual ISFETs in arrays have been observed to have ranges greater than the power supply [6], [8]. This offset can be compensated by hot electron injection [19], UV irradiation [20], and FowlerNordheim tunneling [21]. On chip circuits techniques that do not rely on external equipment, or off-chip instrumentation are preferable. These include adjusting the reference voltage [9], or resetting the floating gate voltage to a fixed voltage biasing before measurements [7], [11]. However, a large reference voltage for mismatch compensation can potentially damage the sensor, and resetting the floating gate could introduce leakage current in sub-micron processes, but also distortion due to temperature dependency.

\section{PH-TIME SENSOR FOR FAST SAMPLING}

If we consider that ISFETs in unmodified CMOS are essentially floating gate devices, and by analysing them in this way the ISFET based pH-Time converter in [12] can be treated as a level-crossing comparator, as shown in Fig. 2. The gates of two transistors are shorted and extended to the top metal layer, with the passivation layer directly on top of this top metal acting as the sensing membrane and forms a capacitor $C_{\text {pass }}$ between metal underneath and electrolyte. Within the electrolyte, the chemical potential $V_{\text {chem }}$ is determined by ion concentration. The electrolyte is also biased at $V_{\text {ref }}$ via a reference electrode. A chemical double layer capacitance, $C_{d l}$, is formed due to the ion distribution profile across the boundary between electrolyte and solid sensing membrane. This capacitance is much larger than the passivation layer capacitance, and can be ignored for further analysis [8], [22]. A control gate, PG, via a MOM (metal oxide metal) capacitor is attached to the floating gate to modulate the floating gate voltage, as shown in Fig. 2.(b).

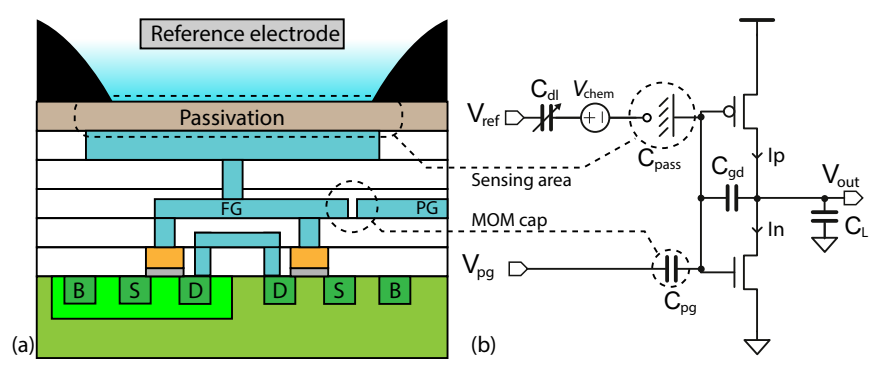

Fig. 2. (a) The cross-section view of an inverter-based pH-time level crossing converter; (b) Schematic of the sensor with voltage inputs and output, and chemical signal source.
The floating gate voltage $V_{F G}$ of the ISFET can be defined as in [8]:

$$
\begin{aligned}
V_{F G}= & \frac{C_{\text {pass }}\left(V_{\text {chem }}+\Delta V_{t h}\right)+C_{p g} V_{p g}+C_{g d} V_{\text {out }}}{C_{F G}} \\
& +\frac{C_{\text {pass }}\left(V_{\text {ref }}\right)+C_{g s P} V_{d d}}{C_{F G}}+k
\end{aligned}
$$

where $C_{\text {pass }}$ is the equivalent capacitance for chemical and passivation interface, $C_{p g}$ and $V_{p g}$ are the capacitance and voltage for the programmable gate, $C_{g d}$ and $V_{\text {out }}$ are the total equivalent gate drain capacitance and drain voltage, $C_{g s P}$ is the gate source capacitance of the PMOS, $C_{F G}$ is the total capacitance seen at the floating gate node [8], $\Delta V_{t h}$ is the sensor offset referred to the reference electrodes due to trapped charge [8], $k$ is the DC term including electrolyte-metal and electrolyte-insulator interface potential, and parasitic influence from source voltage of NMOS. A detailed discussion of these parameters is beyond the scope of this paper and can be found in [23], [24] for floating gate devices, and [22] for electrode electrolyte interface. To simplify the analysis, $C_{g d}$ is assumed to be constant, and the biasing $V_{\text {ref }}$ can be ignored in the following sections if it is shorted to the ground.

The chemical signal through the passivation capacitor, $V_{\text {chem }}$, sensor mismatch, and the control voltage at the programmable gate, $V_{p g}$, are compressed through the capacitive coupling to the floating gate, even with large sensor offset. Hence the readout circuits can recover the chemical signal without saturating the output. However this comes with a penalty of increased circuits noise contribution when referred to the input. According to the literature [8], [25], the noise of transistor is well below the sensor noise and drift. Therefore, the dynamic range of readout can be enhanced with negligible noise increase. Moreover, this floating gate inverter performs level-crossing based comparison on the floating gate via the capacitors, which exhibits lower mismatch and non-linearity.

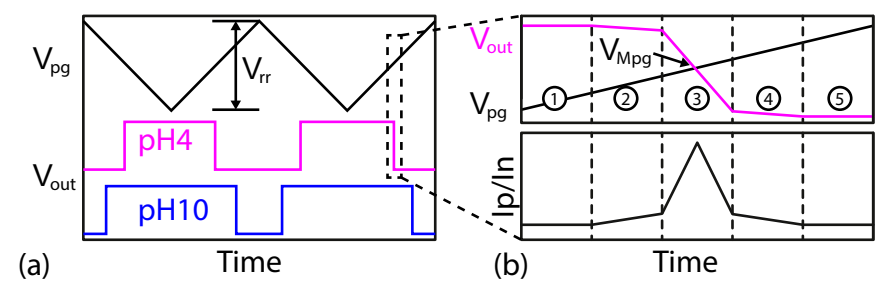

Fig. 3. (a) Illustration of sensor output when a periodical triangle waveform is applied at the PG node; (b) A zoomed view for the output voltage and transistor current during the transition edge.

An illustration of the ISFET inverter output is shown in Fig. 3. With a triangle waveform applied at $V_{p g}$, the inverters output pulsed waveform depending on the switching point of the floating gate voltage. The $\mathrm{pH}$ concentration change induces change in $V_{\text {chem }}$, shifts the switching point and causes different pulse width at the inverter output, as shown in Fig. 3.a. Within the switching transition as shown in Fig. 3.b, the current through transistors are charging or discharging the output capacitor. The propagation delay according to a input step can be estimated by replacing gate voltage with (2) as in [26] 
$C_{L} \frac{d V_{\text {out }}}{d t}=C_{g d}\left(\frac{C_{p g} d V_{p g}}{C_{F G} d t}+\frac{C_{g d} d V_{\text {out }}}{C_{F G} d t}-\frac{d V_{\text {out }}}{d t}\right)+I_{p}-I_{n}$

where $I_{p}$ and $I_{n}$ are the current for PMOS and NMOS receptively, as shown in Fig. 2.b. Without solving this differential equation in different transistor regions, we can simplify it to quasi-steady analysis due to the triangle ramp being much slower than propagation delay of the inverter. With a rising input signal, the operation of the inverter can be simplified to five stages in a zoomed version during transition shown in Fig. 3.b: (1) NMOS off, $V_{d s p}=0$; (2) PMOS in linear region, NMOS in saturation; (3) NMOS and PMOS in saturation; (4) NMOS in linear region, PMOS in saturation; (5) PMOS off, $V_{d s n}=0$. Therefore by assuming $I_{p}=I_{n}$, Eq.(3) has a similar form as Eq.(2). For a given switching point (e.g. $V_{\text {out }}=V d d / 2$ ), the floating gate voltage switching point $V_{M p g}$ can be derived by assuming $I_{p}=I_{n}$ in saturation mode, i.e. phase (3), and it is related to the process parameters and the supply voltage. The threshold of $V_{p g}$ when the output of inverter changes polarity is given by:

$$
V_{M p g}=\frac{\left(V_{M}-k\right) C_{F G}+C_{p a s s} V_{c h e m}+C_{g d} V_{d d} / 2}{C_{p g}}
$$

Therefore the pulse width output can be defined by two transitions of the output, i.e. falling and rising, and transition happens when the programmable gate voltage equals to $V_{M p g}$. By assuming the output is a negative pulse ranging from ground to $V_{d d}$, and input on the programmable gate is a linear triangle signal, the pulse width is given by:

$$
\begin{aligned}
T_{\text {out }} & =\left[T_{1 \rightarrow 0}, T_{0 \rightarrow 1}\right] \\
& =T_{\text {tri }}\left(1-\frac{V_{M p g}}{V r r}\right) \\
& =T_{t r i}\left(1-\frac{\left(V_{M}-k\right) \sum C_{F G}+C_{g d} V_{d d}-C_{g s P} V_{d d}}{V_{r r} C_{p g}}\right. \\
& \left.+\frac{C_{\text {pass }}\left(V_{\text {chem }}+\Delta V_{t h}\right)}{V r r C_{p g}}\right)
\end{aligned}
$$

where $V_{r r}$ is the peak-to-peak voltage of the triangular wave as shown in Fig. 3, $V_{d d}$ is the supply voltage of the inverters. $T_{t r i}$ is the period of the triangle waveform at $V_{p g}$.

It should also be noted that the following condition needs to be met:

$$
\begin{aligned}
\frac{V_{r r} C_{p g}}{C_{F G}} & >V_{d d}-N M_{n}-N M_{p} \\
V_{F G \min } & <N M_{n} \\
V_{F G \max } & >N M_{p}
\end{aligned}
$$

where $N M_{n}$ and $N M_{p}$ are the noise margin values for the inverter [27]. This is to ensure the inverter is able to switch out of noise margin.

\section{A. Offset compensation and signal gain}

CMOS ISFETs suffer a large offset variation, which can be larger than the rail voltage, making on-chip biasing impossible (or extremely challenging). External reference biasing requires extra driving circuits, and adding switches to reset the gate of ISFETs could introduce distortion and leakage. For the proposed scheme, the offset included in the term $\Delta V_{t h}$ can be attenuated and compensated for by using a large swing of the triangle waveform and a suitable capacitor ratio, according to Eq.(5). The voltage swing referred to the floating gate is limited by the maximum voltage swing $\left(V_{r r}\right)$ at the control gate $V_{p g}$, and can be reduced to a smaller value as long as Eq.(6) is fullfilled. With the offset compensated for, the signal gain is given by $T_{t r i} C_{p a s s} /\left(V_{r r} C_{p g}\right)$ for voltage to time conversion, and $\alpha T_{\text {tri }} C_{\text {pass }} /\left(V_{r r} C_{p g}\right)$ for $\mathrm{pH}$ to time conversion with $\mathrm{pH}$ sensitivity of $\alpha m v / p H$.
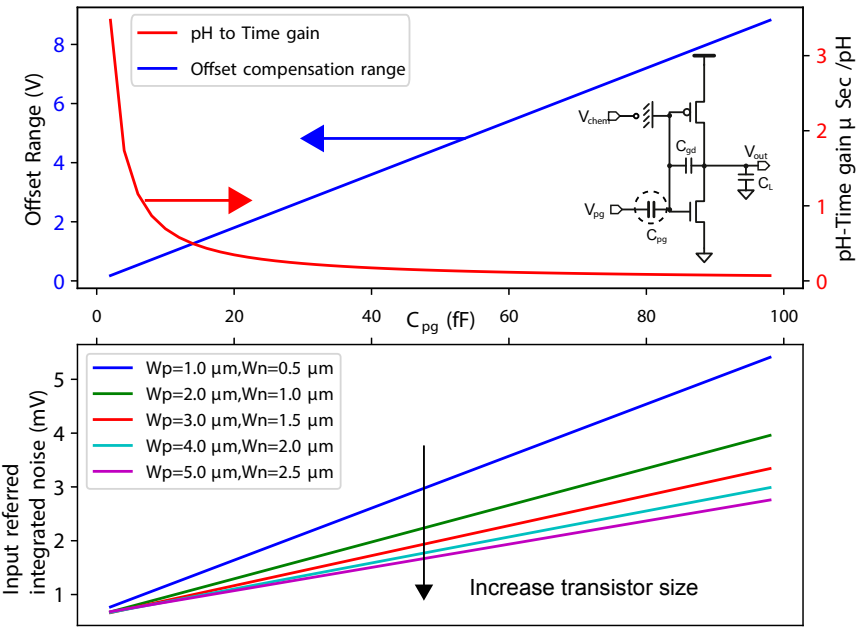

Fig. 4. (a) Offset compensation range and $\mathrm{pH}$-to-time gain change versus the capacitance on the programmable node. (b) Input referred noise change versus different $C_{p g}$ and transistor size.

It can be found that there is design trade-off between the range of compensation and signal gain from chemical sensitivity to pulse output: the larger $C_{p g}$, the larger the offset compensation range, however this would reduce the signal gain due to a small $C_{p a s s} / V_{r r} C_{p g}$. It should also be noted that due to limited resolution of the time to digital converter, the LSB together with the signal gain define the minimum $\mathrm{pH}$ resolution. By assuming the total gate capacitance is $10 \mathrm{fF}$, and sensing membrane is $10 \mathrm{fF}$, the simulated signal gain and offset compensation change versus different $C_{p g}$ is shown in Fig. 4.a. This suggests that by giving an offset variation range an optimum ratio for capacitors should be identified without compromising the $\mathrm{pH}$ sensitivity.

\section{B. Noise and linearity}

During the control signal ramp, the noise accumulated on the output is a stochastic process with noise source from the control signal $\left(V_{n p g}\right)$, the chemical noise $\left(V_{n c h e m}\right)$, and noise from the transistors, as shown in Fig. 5. To solve this explicitly, a stochastic integral from phase 1 to 5 , or an impulse sensitive function should be derived [28]. However the calculation can 


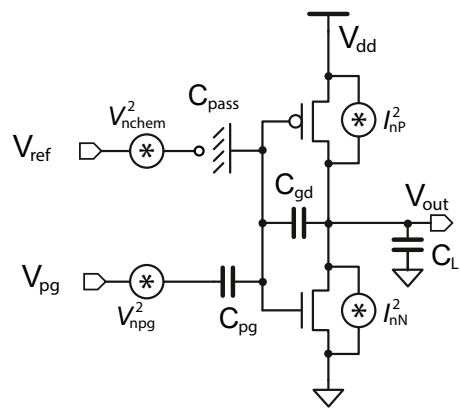

Fig. 5. A simplified noise model of the ISFET inverter.

be simplified by assuming the similar quasi-steady state exists during the switching of the inverters, and steady state noise during the transition dominate the total output phase noise. During this moment, phase 3 in Fig. 3, both transistor are in saturation region, and the entire readout behaves as a capacitively-coupled amplifier with the inverter as the openloop gain. Therefore similar to AC-couple neural amplifiers [29], the total input referred noise to the chemical gate is given by:

$$
\begin{aligned}
\overline{V_{i r n}^{2}} & =\overline{V_{n p g}^{2}} C_{p g} / C_{p a s s} \\
& +\left(\overline{I_{n P}^{2}}+\overline{I_{n N}^{2}}\right) /\left(g_{m P}+g_{m N}\right)^{2} C_{F G} / C_{\text {pass }} \\
& +\overline{V_{n c h e m}^{2}}
\end{aligned}
$$

where $g_{m P}$ and $g_{m N}$ are the transconductance, $I_{n P}^{2}$ and $I_{n N}^{2}$ are the noise sources from transistor including both thermal and flicker noise, $V_{n c h e m}$ is the chemical noise, and $V_{n p g}$ is the noise source from the triangle waveform generator. It can be found within $\mu \mathrm{A}$ spontaneous current as shown in Fig. 3, the noise contribution from transistors are dominated by flicker noise within several $\mathrm{kHz}$ range. The calculated noise level is (482/f) $\mu \mathrm{V} / \sqrt{H z}$, compared to (303/f) $\mu \mathrm{V} / \sqrt{H z}$ in the simulation results shown in Fig. 14. Index $\mathrm{f}$ denotes the frequency at which the noise has been characterised. This is used to benchmark the noise compared to other sensors and the intrinsic MOSFET device noise, instead of using the corner frequency that is dominated by the influence of the chemical noise in CMOS ISFETs.

Compared to a conventional readout, this circuit adds an extra noise source through the control gate, and the input referred noise from transistors are increased due to increased total capacitance seen at the floating gate. However the noise efficiency factor for the transistor noise is improved due to the complementary pair of inverters and duty-cycling the power during sampling. The waveform generator for the control signal is shared by the entire array, and noise can be minimized by using extra power and larger passive devices. The influence of transistor size and $C_{p g}$ for integrated input referred noise without chemical noise is also plotted in Fig. 4(b). Increase of the transistor size reduces the flicker noise, but increase the total floating gate capacitance.

Due to capacitive division shown in Eq.(5), the gain of this readout is linear and mainly limited by the linearity of the waveform generator and the time to digital converter. Design techniques should be considered for a fully on-chip solution to achieve the required linearity and resolution.

\section{Power consumption and supply voltage}

The power consumption of an array of sensor pixels can be estimated by:

$$
P_{\text {array }}=n * F p s *\left(C_{\text {out }} V_{d d}^{2}+C_{p g} V_{r r}^{2}+V_{d d} \int I_{p}, I n\right)
$$

where $n$ is the array size, FPS is the frames per second for the entire array, and $I_{p}$ and $I_{n}$ are the shorting current passing through the transistors as shown in Fig. 3. The shorting current is the current to sustain the operation of both PMOS and NMOS during the transition of charging and discharging the load capacitor. [30]. Both charging and discharging the capacitor and the integration of the short circuits current is dependant on the biasing voltage. By reducing the supply voltage, the transistors in the phase 2-4 will enter weak inversion region. Therefore the overall power consumption increases linearly with the array size and frame sampling speed, and quadratically related to the power supply.
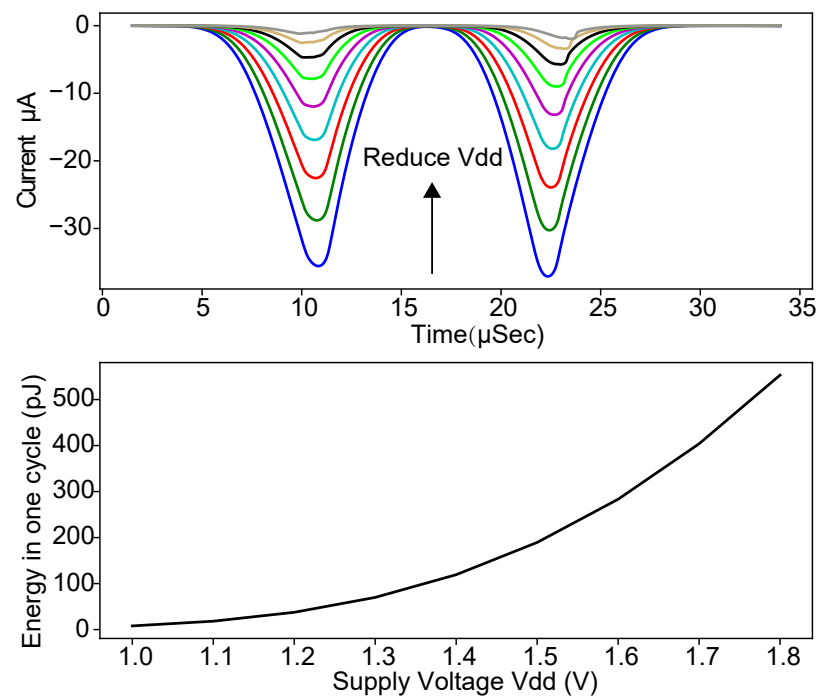

Fig. 6. (a) Simulated current consumption across one sampling cycle versus different supply voltage Vdd, when a triangle waveform with period of $32 \mu \mathrm{S}$ and swing same as the Vdd. (b) Energy consumption within one cycle.

The simulated results for single pixel power consumption is illustrated in Fig. 6 with $1 \mathrm{pF}$ load. With supply voltage decrease from 1.8 to $1 \mathrm{~V}$, the peak shorting current decrease from $37 \mu \mathrm{A}$ to $1.95 \mu \mathrm{A}$, and average energy consumption within one cycle decrease from $553 \mathrm{pJ}$ to $7.992 \mathrm{pJ}$. This spontaneous current also set the thermal noise limit and noise efficiency can be improved in weak-inversion with a reduced supply voltage. In a large-scale sensor array with small transistor sizes and large flicker noise, the power for each pixel can be aggressively reduced to shift the corner frequency of noise characteristic beyond the sampling frequency.

It should be noted however, the energy consumption due to shorting current is related to the period of triangle waveform, the faster the sampling rate, the lower energy per each sample. Therefore the average power consumption due to shorting 
current is independent of the frame rate as long as the quasisteady condition is fulfilled. In addition to the sensor power consumption, the time to digital converter will have a similar relationship between power, speed and supply voltage. Thus for large scale arrays, digital synthesis with power and speed estimation can be used to implement fast sensing systems.

\section{SySTEM IMPLEMENTATION}

\section{A. Design overview}

To demonstrate the proposed scheme and analysis discussed in the previous section, a chemical sensing array is implemented in this work. Design decisions should be made based on the trade-off between different specification, which can be categorized into three groups depending on their priorities:

- Limitation: these includes fundamental design constrains, imposed by the foundry or system requirement, such as capacitor density, pitch size of digital circuits, power supply range.

- Essential: Critical design specification required to enable operation of the sensors, i.e. the threshold voltage variation range for the sensors.

- Performance: Specification to enhance the system performance, such as noise, sensitivity and linearity of sensors, signal gain, dynamic range of the converters.

In this design, the targeted sensing system includes $32 \times 32$ pixels with maximum $5 \mathrm{k}$ sampling rate. The implemented system should be within $1 \times 1 \mathrm{~mm}^{2}$ silicon area in a typical $0.18 \mu \mathrm{m}$ CMOS technology with the core supply voltage of $1.8 \mathrm{~V}$. These limitations are the starting point for the design process.

\section{B. Sensor pixel and array}

Given the area constrains, the pixels size has to be smaller than $30 \times 30 \mu \mathrm{m}^{2}$, and was designed at $26 \times 26 \mu \mathrm{m}^{2}$ to leave some margin for the peripheral circuits and interconnection. From discussion in section III, the chemical sensing area should be maximized to introduce a larger $C_{\text {pass }}$, which is preferred for noise minimizing and signal amplification. To reduce the coupling effect between adjacent sensors, a $75 \%$ filling rate is chosen to leave a $8 \mu \mathrm{m}$ gap between sensors. This sets the sensing membrane, defined by the top layer metal (M6) plate, to $22 \times 22 \mu \mathrm{m}^{2}$, and gives a $C_{\text {pass }}$ around $10 \mathrm{fF}$ based on process documents and [8].

According to previous studies [6], [8], CMOS ISFETs exhibit large threshold offset up to $\pm 10 \mathrm{~V}$. To compensate a target variation of $\pm 5 \mathrm{~V}$, the capacitance of the programmable gate $C_{p g}$ should be set to around $56 \mathrm{fF}$ by using the Eq.(2) and Fig. 4. This capacitor was implemented in a MOM (metal oxide metal) structure with size of $10 \mu \mathrm{m} \times 10 \mu \mathrm{m}$ considering the process technology and area limitation.

From the Eq.(7) and Fig. 4, the transistor size should be minimized to reduce the capacitor ratio for input referred noise, however, not too small to avoid transistor flicker noise exceeding the intrinsic low frequency noise and drift [8] of the sensors. The contribution of flicker noise of transistors towards input referred noise can be simulated as shown in
TABLE I

SUMMARY OF PIXEL IMPLEMENTATION

\begin{tabular}{|c|c|c|}
\hline Specification & Normal & Diode \\
\hline M6 size & $9 \times 9$ & $22 \times 22$ \\
\hline M5 size & $20 \times 20$ & N/A \\
\hline $\begin{array}{l}C_{p g} \\
\text { Sub-array size }\end{array}$ & \multicolumn{2}{|c|}{$\begin{array}{c}10 \mathrm{fF}, 10 \times 10 \mu \mathrm{m}^{2} \mathrm{M} \\
16 \times 32\end{array}$} \\
\hline
\end{tabular}

Fig. 4. The drift and low frequency noise limits of the sensor can be defined according to [8], [9], [13], [16]. It can be found the noise contribution from minimum transistors of this technology is much smaller than the drift rate reported. Therefore a standard inverter with PMOS size of 930n/180n and NMOS size of 220n/180n is placed underneath the sensing metal layer, with gates connected to sensing area with a metal stack.

During physical layout, an antenna violation was found at the gate of complimentary pairs, due to a large top thick metal plate connected to them. This can be resolved by connecting a tie-down minimum size $5 \mathrm{~V}$ substrate diode to the floating gate node. This diode also introduces a high resistance path to the ground, and in theory can suppress the offset in a similar manner as a reset switch. During power up the chip and switching of the inverter, the floating gate steady state voltage can be passively defined by this diode without any active biasing. In addition to diode pixels, half of the array $(16 \times 32)$ was based on a similar pixel design with combinational sensing area of $9 \times 9 \mu \mathrm{m}^{2}$ of top metal (M6) and $20 \times 20 \mu \mathrm{m}^{2}$ of M-1 (M5). This large M5 plate is to increase the sensing area and consequently $C_{\text {pass }}$ This configuration reduces the effective $C_{\text {pass }}$ and affects the noise and signal gain performance accordingly. A simplified illustration of both pixels are shown in Fig. 7, with pixels specification listed in Table.I

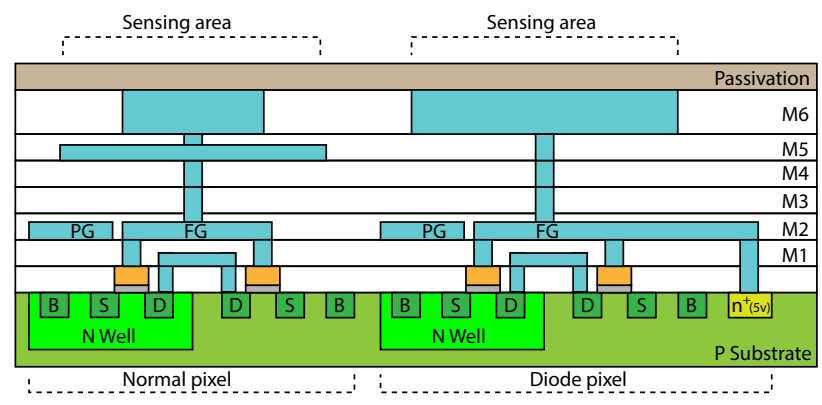

Fig. 7. Two types of pixel implemented in the $32 \times 32$ array. Left: the normal pixel with a smaller M6 and a larger M5 metal plate as the sensing area; Right: the diode pixel with a larger top metal M6 as the sensing area. The interdigitated MOM structure is simplified to a single dual-plate capacitor.

The inverter output is connected to a complimentary switch with a minimum size. The entire array has $32 \times 32$ sensors with output from same row shorted to the input of the timeto-digital converter. 


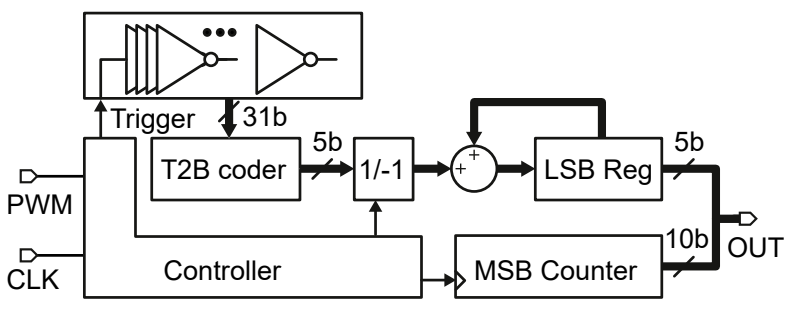

Fig. 8. Diagram of the proposed TDC structure.

\section{Asynchronous TDC}

Previous TDCs used in ISFET interfaces rely on a synchronised triggering signal [6], [7], [31]. However since the level crossing could be triggered at arbitrary time, the transition of the pixel output cannot be synchronised to a clock. Using a fully delay line based configuration requires a relatively large silicon area and power consumption. In this work, an asynchronous TDC for capturing the ISFET output is proposed, as shown in Fig. 8

1) Resolution requirement: The period of triangle waveform $T_{t r i}$ can be chosen based on bandwidth of on-chip or offchip waveform generator, and the sampling rate requirement for the entire array. With a target of $5 \mathrm{k}$ frames per second for this $32 \times 32$ system, the column-wise sampling rate should be at least $160 \mathrm{k}$ per column, which is the maximum input range of the TDC.

Moreover, according to Eq. (5), the output time signal reflects the sensor offset in the form of different pulse width. Therefore the range of this time signal variation should also be smaller than the sampling period, as shown below:

$$
\begin{array}{r}
\text { Range }_{T D C} \in\left[t_{0}+T_{t r i} \frac{C_{p a s s} V_{t h-}}{V_{r r} C_{p g}},\right. \\
\left.t_{0}+T_{t r i} \frac{C_{p a s s} V_{t h+}}{V_{r r} C_{p g}}\right]
\end{array}
$$

Where

$$
\begin{aligned}
t_{0}= & T_{t r i}\left(1-\frac{\left(V_{M}-k\right) C_{F G}+C_{g d} V_{d d}}{V_{r r} C_{p g}}\right. \\
& \left.+\frac{C_{g s P} V_{d d}}{V_{r r} C_{p g}}\right)
\end{aligned}
$$

$V_{t h-}$ and $V_{t h+}$ are the minimum and maximum threshold mismatch when referred to the reference electrode. Since the target of the compensation range for input-referred mismatch is $\pm 5 \mathrm{~V}$, therefore peak-to-peak value of output pulse width is $6.2 \mu \mathrm{s}$, which is smaller than the sampling period for each column. The nominal pulse width given by the $t_{0}$ can be tuned by changing supply voltage of the inverters, or the swing voltage of the triangle waveform as shown in Eq.(9), such that the full dynamic range of the TDC can be utilized even with extreme mismatch value. It should be noted that the reference voltage is kept at $0 \mathrm{~V}$ in Eq.(9), and can be changed to tune nominal pulse width following similar steps from Eq.(2) to Eq.(9)

With predefined $C_{p a s s}, C_{p g}, C_{F G}$, and supply voltage of the sensors, the signal gain of sensors can be derived based on Eq.(5). The LSB of time to digital converters determines



Fig. 9. Timing diagram of the TDC.

the $\mathrm{pH}$ resolution. The sensitivity requirement of $\mathrm{pH}$ can be converted to the time resolution of the time to digital converters, by using Eq.(5). The required TDC resolution, LSB, should be defined by:

$$
L S B_{T D C}=\frac{\gamma p H_{m i n} C_{p a s s} T_{t r i}}{V_{r r} C_{p g}}
$$

where $\gamma$ is the coefficient for Nernstian sensitivity [13]. The maximum range of TDC is $T_{t r i}$. To achieve $0.1 \mathrm{pH}$ resolution with the ideal Nerstian sensitivity of $59 \mathrm{mV} / \mathrm{pH}$, the LSB of TDC is $3.66 \mathrm{~ns}$.

By combining Eq.(9) and (10), the dynamic range of the TDC should be larger than 12-bits, when a mismatch range of $\pm 5 \mathrm{~V}$ and $0.1 \mathrm{pH}$ resolution is required. Considering linearity of the TDC and possible offset of the transistors, a 15-bit configuration was chosen.

2) Course and Fine conversion: To reduce the total area, a coarse-fine structure is used to implement the multi-bit TDC [31]. In this design a 10-bit coarse converter has been designed using a 10-bit asynchronous counter to reduce the total number of logic gates, and the fine converter was based on a 31stage current starved delay line. The delay is controlled by a global voltage bias, generated by an external current source. These enable a wide-tuning range for the LSB of the TDC from $180 \mathrm{ps}$ to $9.5 \mathrm{~ns}$ with the clock frequency for the counter ranging from $3.2 \mathrm{MHz}$ to $164.8 \mathrm{MHz}$. The mismatch between the LSB of the coarse converter and the full range of the fine converter, is adjusted externally by tuning the reference current input or the clock of the counter.

3) Asychronous Logic: Since the edge of the PWM waveform is not aligned to the clock synchronization, the control logic of the TDC will detect and divide the PWM signal to three parts and initialise conversion by using either coarse or fine conversion. A detailed conversion timing diagram is shown in Fig. 9.

i Once a rising edge of PWM is detected at time $a$, the controller will pass the rising edge to the delay line and initialise the edge propagation; the screen shot of the delay line status, i.e. the timing information, will be captured by the next clock rising edge at $b$.

ii From $b$ the counter will be enabled and increments every rising edge of clock, until PWM falls.

iii The falling edge of the PWM at time of $c$ will be inverted and fed into delay line again; the delay line value between the PWM falling edge $c$ and immediate next rising edge $d$ of the clock will be captured and subtracted from the 
pre-stored $T_{a \rightarrow b}$;

iv the value stored in the MSB counter and LSB register $\left(T_{a \rightarrow b}-T_{c \rightarrow d}\right)$ will be combined to give valid output results of 16 bits (10-bit unsigned and 6-bit signed).

4) Scalability of the TDC: The proposed inverter delay line can be replaced by a standard delay line with minimum sized inverters if a smaller and fixed LSB value is desired. This fully digital implementation can be easily scaled according to different sampling speed and area requirements. The frame sampling speed is limited by the dynamic range of the TDC, also related to frequency of triangular signal:

$$
\begin{aligned}
\text { Fps } & =\frac{n_{\text {row }}}{T_{\text {trig }}} \\
& \leq \frac{n_{\text {row }}}{L S B \times D R}
\end{aligned}
$$

where $n_{\text {row }}$ is the number of row elements, $D R$ is the dynamic range of TDC and is related to the number of bits, and $L S B$ is the minimum propagation delay in the delay line, and can be scaled down to picoseconds when advanced technology is used. This suggests that a sampling rate of $\approx \mathrm{kFPS}$ can be easily achieved in large arrays. Moreover the physical implementation of the TDC is only limited by the pitch size of the digital core cells; this makes a column-wise implementation feasible even for micron-scale pixels. Last but not least, with fully digital implementation, a low supply voltage is possible to power up the TDC, and significantly reduce the power consumption. The minimum supply voltage is limited by the unit delay of the inverter chains, which can be reduced to half the power supply voltage in modern CMOS technologies.

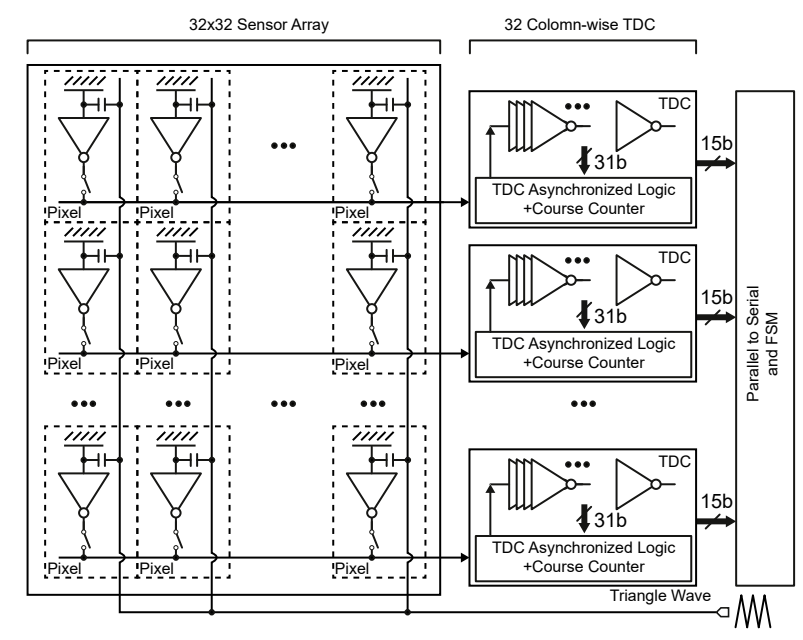

Fig. 10. Overview of the sensing system with inverter based sensing pixels and column-wise asynchronous coarse-fine TDC.

\section{Overall system}

The overall system diagram is shown in Fig. 10, which is divided into three parts: (1) a $32 \times 32$ sensor array with in-pixel digitization generating a 32-bit digital pulse width output; (2) 32 column-wise 15-bit coarse-fine time to digital converters that directly convert the pulse widths to parallel digital output;

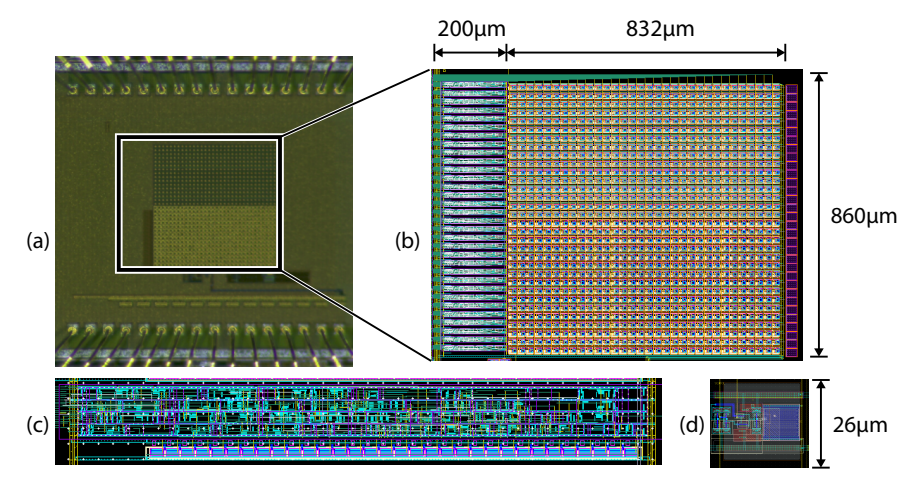

Fig. 11. (a) Micro-photograph of the fabricated array and test circuits; (b) Layout of the entire system with size of $860 \times 1032 \mu \mathrm{m}^{2}$; (c) TDC with size of $26 \times 200 \mu \mathrm{m}^{2}$, d) Sensor pixel with size of $26 \times 26 \mu \mathrm{m}^{2}$

(3) $32 \times 15$-bit digital output are fed in parallel to top level digital controller for a parallel-in-serial-out interface. The top level logic was shared with other test circuits and synthesised into a single block. It also includes a SPI link for single pixel results polling and configuration on the sampling speed.

The system is implemented in a typical $0.18 \mu \mathrm{m}$ CMOS technology, with the layout and micro-photograph shown in Fig. 11. The height of pixel and TDC are aligned for convenient synthesis and connection.

\section{RESULTS AND DISCUSSION}

The fabricated silicon chip was firstly wire-bonded into a ceramic package. The bonding wires and pads were encapsulated by using ELECTROLUBE potting epoxy ER2223. A plastic tube was mounted on top of the encapsulated chip as the chemical chamber, by using silicone based glue. $\mathrm{An} \mathrm{Ag} / \mathrm{AgCl}$ wire was attached to the tube and acting as the reference electrode. The electrode was biased at a fixed voltage and immersed into the solution.

To establish data communication and configure the chip settings, a DE1SOC development board was used as the datatransmission hub. The serial link from the sensors system was acknowledged by the FPGA and pushed into memory FIFO, the operating system in the DE1SOC fetched the data via the data bus and sent to the computer via the Ethernet port. A shield PCB was designed to accommodate the sensor chip, and also provided voltage biasing for the delay line. The FPGA also generates a synchronization signal depending on the chip serial link, and triggers the waveform generator AFG3102 to generate the required triangle waveform. The overall system is illustrated in Fig. 12.a with a photograph of the test platform shown in Fig. 12.b.

Due to speed limitation on the DE1SOC board pin IO (with extra resistance and ESD diodes), the maximum speed for reliable clock and data transmitting was found to be $50 \mathrm{MHz}$. Therefore the system sampling rate was fixed to $1 \mathrm{kFPS}$ in this measurement, compared to $5 \mathrm{kFPS}$ in simulation. Under $1 \mathrm{kFPS}$ sampling speed, the power consumption measured for sensor array, digital circuits (TDC and FSM), and pads (driving $2 \mathrm{pF}$ load) were $2.47,2.60$, and $1.2 \mathrm{~mA}$ current. This gave total power consumption of $11.286 \mathrm{~mW}$. 


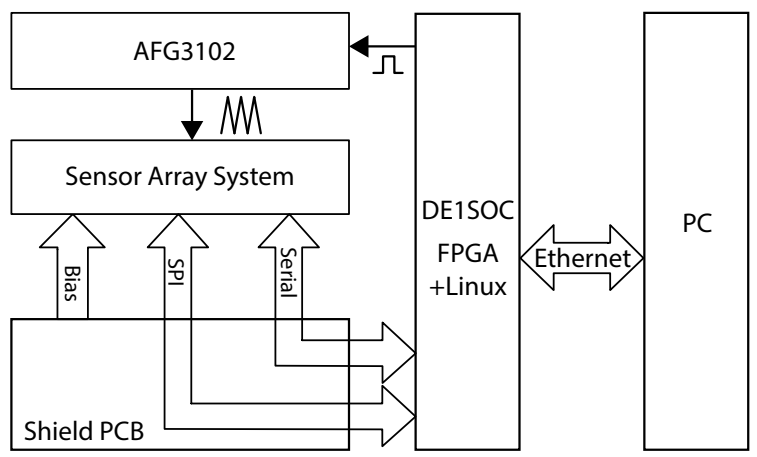

(a)

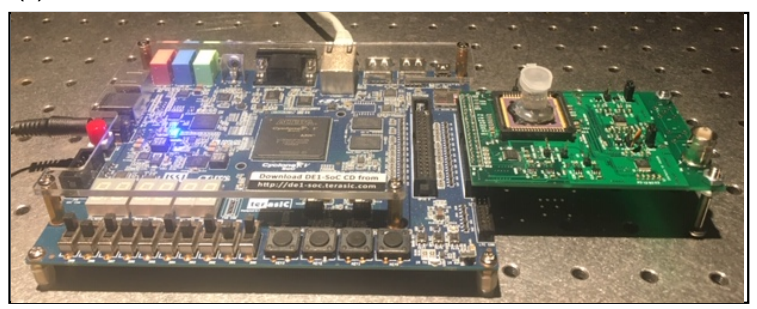

(b)

Fig. 12. Experimental setup for the measurement: (a) Overall diagram of the test system; (b) The ISFET sensor chip mounted on a shield-PCB, and the $\mathrm{PCB}$ is connected to the DE1SOC development board directly.
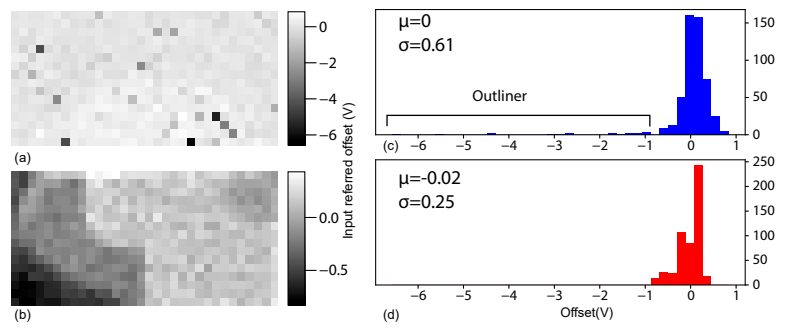

Fig. 13. Offset across the entire array: (a) Normal pixel, (b) diode pixel, (c) histogram of normal pixel, (d) histogram of diode pixel.

To identify the voltage to time conversion gain from chemical environment to time domain, a step change of $200 \mathrm{mV}$ was applied on the reference electrodes, resulting in 457 LSB (normal pixel) and 764 LSB (diode pixel) change for the TDC output. This value gave a ratio between reference voltage to digital output at 2.28 LSB per $\mathrm{mV}$ for normal pixels, and 3.82 LSB per $\mathrm{mV}$ for diode pixels, and was used to normalize between $\mathrm{pH}$, voltage, and time output in the following analysis.

\section{A. Sensor offset}

The array was measured with a reference electrode biased at $0 \mathrm{~V}$. The average pulse width is $19800 \mathrm{LSBs}$, and maximum variation of $13500 \mathrm{LSBs}$. This number is the digital equivalents of the pulse width time signal, ranging from 0 to 32767 for the $15 \mathrm{~b}$ TDC. This offset is then referred back to the input, i.e. the reference electrode, giving standard deviation of offset $0.61 \mathrm{~V}$ for normal pixels and $0.25 \mathrm{~V}$ for diode pixels. The distribution of the offset of the entire $32 \times 32$ array is divided depending on the pixel type and is shown in Fig. 13. These results suggest that the offset is mainly due to trapped charge in the passivation not the floating gate [8]. Moreover the diode pixels still exhibit offset compared to normal pixels, therefore

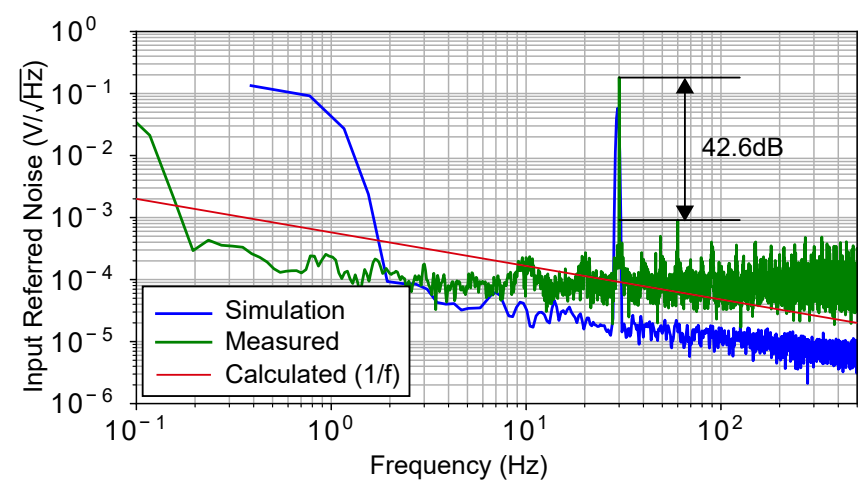

Fig. 14. A comparison of analytical, simulated, and measured results for noise and linearity.

tie-down diodes can not fully eliminate the offset by setting the floating gate voltage, due to the diode mismatch.

The mismatch across sensors for the implemented arrays were much smaller than the other CMOS ISFETs reported [6], [8]. This also indicated that our mismatch range (simulated \pm 5 , measured \pm 7 ) was over-engineered with much larger $C_{p g}$ than the necessary value. It can be reduced to improve the signal gain of pH-sensitivty, as shown in Fig. 4.

\section{B. Noise and linearity}

It is impossible to generate a sinusoid-like chemical signal for noise measurement, therefore, an electrical sinusoid waveform with amplitude of $200 \mathrm{mV}$ and frequency of $30 \mathrm{~Hz}$ is generated to emulate of $\mathrm{pH}$ change. The output value was then back calculated to the reference electrode to get the signal gain from input voltage to output digital signal, and then further compared to the $\mathrm{pH}$-sensitvity such that $\mathrm{pH}$ related noise and linearity can be derived. Fig. 14 shows the spectrum analysis from the measured output (green), simulated results before the data converter (blue), and analytical results using operation point value during switching (red). In the simulation setup the same biasing and transistor size were used, and the programmable gate is driven by a triangle signal with bandlimited noise source of $1 \mathrm{mV}$ within $1 \mathrm{MHz}$ bandwidth.

Compared to simulation results, the measured results shows an input referred white noise level of $100 \mu / f \mathrm{~V} / \sqrt{\mathrm{Hz}}$, and integrated noise from $2 \mathrm{~Hz}$ to $500 \mathrm{~Hz}$ is $2.4 \mathrm{mV}$, indicating a minimum $\mathrm{pH}$ related noise level of $0.08 \mathrm{pH}$. The corner frequency between thermal and flicker noise is around $3 \mathrm{~Hz}$. However it is arguable whether it is purely flicker noise or also affected by the drift [8]. The SFDR was $42.6 \mathrm{~dB}$, mainly due to mismatch of LSBs or nonlinear relation between reference voltage and chemical capacitance.

The discrepancy of noise level and non-linearity are because of: (1) noise from the waveform generator are larger than the estimation ( $1 \mathrm{mV}$ within $1 \mathrm{MHz})$; (2) the quantization noise, mismatch of the data converter; (3) chemical noise due to reference electrode, electrolyte-insulator membrane and distortion introduced in the double layer and site-binding model. Detailed analysis of the chemical noise is beyond the scope of this paper. However the measured noise level is comparable to other results reported [5], [6], [8]. 

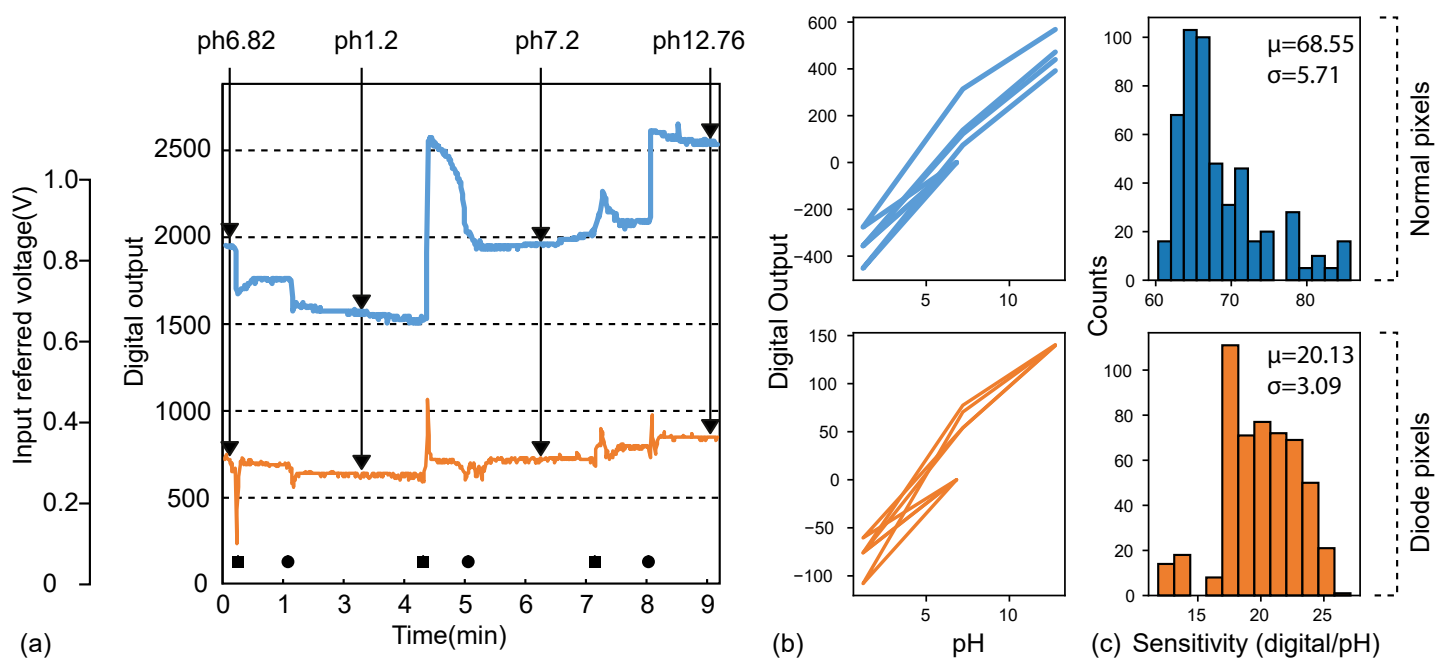

Fig. 15. pH sensitivity characterization. Normal pixels results are shown in blue, and diode pixel results are shown in yellow. Shown is (a) Transient pH response with acid and base added at time labelled as squre, and active convection labelled as round; (b) Extracted pH sensitivity for 4 sensors in the normal array and diode array; (c) Histogram of $\mathrm{pH}$ sensitivity across entire array



Fig. 16. Long term drift analysis, a) drift for normal sensors, b) diode pixels.

\section{Long term stability and drift}

The long term drift for the entire sensor array was characterized in a $\mathrm{pH} 7$ buffer solution by biasing the reference electrode at $0 \mathrm{~V}$, and one hour recording was taken after a tenminutes settle-in period. The recorded data was decimated to 4 samples per seconds, and results for 4 sensors, 2 normal pixel and 2 diode pixels are shown in Fig. 16. The normal pixels exhibited drift of $250 \mathrm{mV}$ in one hour, and diode pixel drifted $60 \mathrm{mV}$ per hour. The measured results were comparable to other CMOS ISFETs with un-modified sensing membrane, however the reduced drift seen from all diode pixels are possibly affected by the leakage current through the floating gate.

\section{D. $p H$ sensitivity}

To observe the short time and long time $\mathrm{pH}$ response and diffusion effect of the sensors, a pH 10 buffer solution of $2 \mathrm{ml}$ was used, and various amount of acid (hydrochloride) and base (sodium hydroxyl) was dropped into the buffer solution to change the $\mathrm{pH}$. The sensor array measured chemical response in real-time, and a Sentron $\mathrm{pH}$ meter was used to measure the $\mathrm{pH}$ value at specific point as a reference. The $\mathrm{pH}$ response of a normal pixel and the diode pixel are shown in Fig. 15.a. The acid or base was added at time marked by square, and one minute later, the solution was stirred to force the convection (circle), then the $\mathrm{pH}$ read from Sentron was taken another minute later (arrow).

The digital output gives $\mathrm{pH}$ sensitivity in terms of LSBs. With relationship between $V_{\text {chem }}$ and digital output acquired in previous step voltage sweep, this value can be converted with unit of $\mathrm{mV} / \mathrm{pH}$. These confirmed the $\mathrm{pH}$-sensitivity of both pixels, but also illustrated the different dynamic response of the two sensors: (1) both sensors responded to the $\mathrm{pH}$ change sharply, this is due to acid or base was dropped into the chemical chamber; (2) it took minutes for sensor to settle down, this could be due to diffusion, slow response of the sensing membrane or the drift [22]; (3) diode sensors shows high-pass characteristics for the chemical signal after the fastresponse, and settled down with reduced sensitivity compared to the normal pixel. This is due to the leakage current of the diode.

It should be noted that the 3rd phenomenon is also confirmed by a step change on the biasing voltage as mentioned before, and similar settling effect was observed. This suggested that the tie-down diode could introduce large distortion and sensitivity reduction for ISFETs sensors, depending on different process parameters and initial biasing condition of the substrate. Moreover drift and hysteresis of the sensor also degraded the sensor performance.

The output was then plotted against the $\mathrm{pH}$ measured by the $\mathrm{pH}$ probe, as shown in Fig. 15.b, with histogram of $\mathrm{pH}$ sensitivity across all pixels within the $32 \times 32$ array plotted in Fig. 15.c. An average $\mathrm{pH}$ sensitivity of $70 \mathrm{LSBs} / \mathrm{pH}$, or $30 \mathrm{mV} / \mathrm{pH}$ was achieved for normal pixels, and $20 \mathrm{LSBs} / \mathrm{pH}$ or $5.2 \mathrm{mV} / \mathrm{pH}$ for diode pixels.

\section{E. Fast chemical imaging}

To observe fast response of the sensor system, a base (sodium hydroxyl) solution of $0.5 \mathrm{ml}$ was added into the buffer solution. The $\mathrm{pH}$ response of the entire array was recorded simultaneously with $1 \mathrm{kFPS}$. The images taken at different time stamps are shown in Fig. 17, from $1 \mathrm{~ms}$ to $4 \mathrm{~s}$. Part of the 


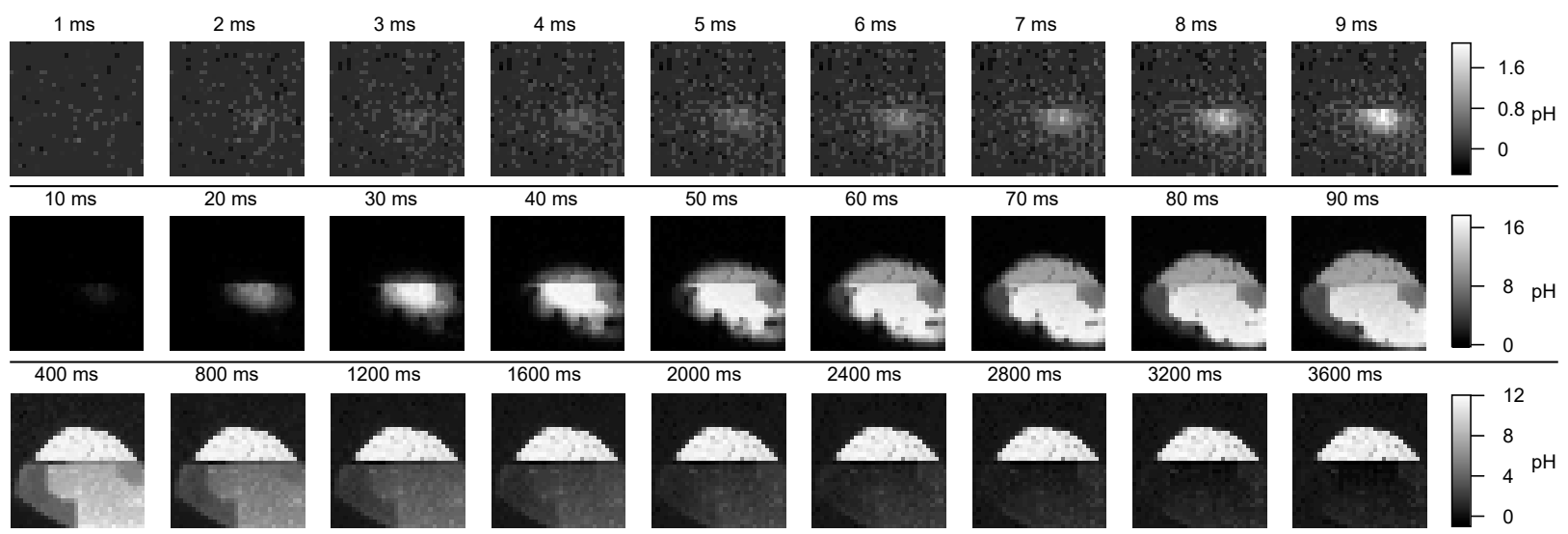

Fig. 17. Fast chemical imaging within a four-second window.

TABLE II

COMPARISON OF CMOS ISFET SENSING ARRAYS

\begin{tabular}{|c|c|c|c|c|c|c|c|c|}
\hline Specification & & This work & [10] & [7] & [11] & [6] & [5] & [17] \\
\hline Process & {$[\mu \mathrm{m}]$} & 0.18 & 0.35 & 0.18 & 0.35 & 0.35 & 0.35 & 0.18 \\
\hline Supply & {$[\mathrm{V}]$} & 1.8 & 3.3 & 3.3 & 3.3 & 3.3 & 3.3 & 3.3 \\
\hline \multicolumn{2}{|c|}{ Offset compensation } & \pm 7 & N/A & Yes & Yes & $1 \mathrm{~V}$ & Yes & Yes \\
\hline Resolution & {$[\mathrm{pH}]$} & 0.013 & N/A & $0.1-0.025$ & 0.06 & 0.019 & 0.1 & N/A \\
\hline Noise & {$[\mathrm{pH}]$} & 0.08 & N/A & N/A & 0.007 & 0.019 & 0.101 & N/A \\
\hline Noise bandwidth & {$[\mathrm{Hz}]$} & $2-500$ & N/A & N/A & $2 m-3.5$ & N/A & 1 & N/A \\
\hline Sensitivity & {$[/ \mathrm{pH}]$} & $30 \mathrm{mV}$ & $20 \mathrm{mV}$ & $26.2 \mathrm{mV}$ & $42 \mathrm{mV}$ & $39.3 \mathrm{mV}$ & $1.03 \mu \mathrm{A}$ & N/A \\
\hline Drift & {$[\mathrm{mV} / \mathrm{s}]$} & $0.7 / 0.2$ & N/A & $0.1-0.025$ & 0.21 & 0.1 & N/A & N/A \\
\hline Linearity & {$[\mathrm{dB}]$} & 42.6 & N/A & N/A & N/A & N/A & N/A & N/A \\
\hline Pixel power & {$[\mu \mathrm{W}]$} & $0.01^{a}$ & 16 & N/A & 0.6 & Varying & $0-200$ & Varying \\
\hline Sensor size & {$\left[\mu \mathrm{m}^{2}\right]$} & $22 \times 22$ & N/A & 22.3 & N/A & N/A & $6.5 \times 7.8$ & N/A \\
\hline Pixel size & {$\left[\mu m^{2}\right]$} & $26 \times 26$ & $10.2 \times 10.2$ & $10 \times 10$ & $50 \times 50$ & $37 \times 31$ & $6.5 \times 7.8$ & $12.5 \times 10$ \\
\hline Array size & & $32 \times 32$ & $64 \times 64$ & $64 \times 64$ & $32 \times 32$ & $78 \times 56$ & $64 \times 200$ & $128 \times 128$ \\
\hline Entire area & {$\left[\mathrm{mm}^{2}\right]$} & 0.9 & 0.49 & 12.5 & 6.3 & 8 & 1.9 & 5.72 \\
\hline Frame rate & [FPS] & $5 \mathrm{~K}^{b} / 1 \mathrm{~K}$ & 100 & 1250 & 9.3 & 0.75 & 7.8 & 7800 \\
\hline Total power & {$[\mathrm{mW}]$} & 11.286@1K & N/A & 32@100 & 10.12@9.3 & 7.5 & N/A & N/A \\
\hline
\end{tabular}

${ }^{a}$ Simulated average power consumption per 1 sample @ $5 \mathrm{~K} ;{ }^{b}$ Simulated;

system was covered by encapsulation, which was intended to form a small chamber to reduce the convection.

A random response pattern was recorded at the first few $\mathrm{ms}$, this is possibly due to forced convection during solution dropping and random diffusion pattern, then a slow (at time scale $10 \mathrm{~ms}$ ) diffusion-like process happened for both types of sensors, the diode sensor exhibited higher short-time sensitivity, possibly due to larger chemical area thus higher $\mathrm{pH}-$ to-Time gain. Around $100 \mathrm{~ms}$ of time, both sensors reached the highest output, i.e. a pH peak. After that the diode pixels exhibited similar settle-down effect with time constant of few seconds.

\section{CONCLUSION}

In this paper, we provided a detailed analysis for $\mathrm{pH}$-Time ISFET sensors with a level-crossing based time-domain readout. The system performance and comparison with state-ofthe-art is summarized in Table II. Compared to other readout circuits, this scheme enabled fast chemical sampling, high linearity, large offset compensation, simple data conversion requirement and acceptable noise level without high power budget. By optimizing the design parameters, the chemical signal can be amplified and output in the form of a digital pulse width with minimal noise and distortion. A $32 \times 32$ ISFET chemical sensing array with asynchronous column-wise time-to-digital converters was designed and implemented in a $0.18 \mu \mathrm{m}$ CMOS process to validate the sensor and system. Half of the sensors have a tie down diode connected to the floating gate to resolve DRC violations and experiment a passive offset compensation scheme.

Measured results show that the offset variation across the entire array was $6 \mathrm{~V}$ with standard deviation of $0.6 \mathrm{~V}$ and were compensated for by using the proposed system. The $\mathrm{pH}$ sensitivity was measured with average of $30 \mathrm{mV} / \mathrm{pH}$ for normal pixels and $5.2 \mathrm{mV} / \mathrm{pH}$ for diode pixels, this gives minimum $\mathrm{pH}$ resolution of $0.013 \mathrm{pH}$ defined by the resolution 
of TDC. The input-referred integrated noise from $2 \mathrm{~Hz}$ to $500 \mathrm{~Hz}$ is $2.4 \mathrm{mV}$, indicating a $\mathrm{pH}$ noise level of $0.08 \mathrm{pH}$. Long term drift was characterized at $250 \mathrm{mV}$ per hour for normal pixels and $60 \mathrm{mV}$ for diode pixels. Fast chemical imaging was achieved with $1 \mathrm{~K}$ frame per second with total power consumption of $6.27 \mathrm{~mA}$. The measured results also indicate that diode connected floating gate may introduce highpass characteristics for chemical signal change, and is not acceptable for long-term chemical sensing depending on the process parameters such as leakage current.

In conclusion, with direct $\mathrm{pH}$-to-Time conversion and a digital pulse width output, the sensor interface can be greatly simplified. There exists a design trade-off between offset compensation, $\mathrm{pH}$ sensitivity, noise, and power consumption. Due to its mostly digital nature, the proposed sensing scheme enables ultra-fast fully synthesisable chemical sensing systems with feature size scalability down to deep sub-micron technology.

\section{ACKNOWLEDGEMENT}

The authors would like to thank Prof. Tor Sverre Lande, Dr Lieuwe Leene and Mr Alan Bannon for their help on technical discussion and manuscript presentation.

\section{REFERENCES}

[1] P. M. Levine, P. Gong, R. Levicky, and K. L. Shepard, "Active cmos sensor array for electrochemical biomolecular detection," IEEE Journal of Solid-State Circuits, vol. 43, no. 8, pp. 1859-1871, 2008

[2] J. Rothberg, W. Hinz, T. Rearick, J. Schultz, W. Mileski, M. Davey, J. Leamon, K. Johnson, M. Milgrew, M. Edwards et al., "An integrated semiconductor device enabling non-optical genome sequencing," Nature, vol. 475, no. 7356, pp. 348-352, 2011.

[3] J. J. Jun, N. A. Steinmetz, J. H. Siegle, D. J. Denman, M. Bauza, B. Barbarits, A. K. Lee, C. A. Anastassiou, A. Andrei, Ç. Aydın et al., "Fully integrated silicon probes for high-density recording of neural activity," Nature, vol. 551, no. 7679, p. 232, 2017.

[4] D. Garner, H. Bai, P. Georgiou, T. Constandinou, S. Reed, L. Shepherd, W. Wong, K. Lim, and C. Toumazou, "A multichannel dna soc for rapid point-of-care gene detection," in Solid-State Circuits Conference Digest of Technical Papers (ISSCC), 2010 IEEE International. IEEE, 2010, pp. 492-493.

[5] N. Miscourides, L.-S. Yu, J. Rodriguez-Manzano, and P. Georgiou, "A $12.8 \mathrm{k}$ current-mode velocity-saturation isfet array for on-chip real-time dna detection," IEEE transactions on biomedical circuits and systems, no. 99, pp. 1-13, 2018.

[6] N. Moser, J. Rodriguez-Manzano, T. S. Lande, and P. Georgiou, "A scalable isfet sensing and memory array with sensor auto-calibration for on-chip real-time dna detection," IEEE transactions on biomedical circuits and systems, vol. 12, no. 2, pp. 390-401, 2018.

[7] X. Huang, H. Yu, X. Liu, Y. Jiang, M. Yan, and D. Wu, "A dualmode large-arrayed cmos isfet sensor for accurate and high-throughput ph sensing in biomedical diagnosis," IEEE Transactions on Biomedical Engineering, vol. 62, no. 9, pp. 2224-2233, 2015.

[8] Y. Liu, P. Georgiou, T. Prodromakis, T. Constandinou, and C. Toumazou, "An extended cmos isfet model incorporating the physical design geometry and the effects on performance and offset variation," Electron Devices, IEEE Transactions on, vol. 58, no. 12, pp. 4414-4422, 2011.

[9] N. Miscourides and P. Georgiou, "Impact of technology scaling on isfet performance for genetic sequencing," IEEE Sensors Journal, vol. 15 no. 4, pp. 2219-2226, 2015.

[10] B. Nemeth, M. S. Piechocinski, and D. R. Cumming, "High-resolution real-time ion-camera system using a cmos-based chemical sensor array for proton imaging," Sensors and Actuators B: Chemical, vol. 171, pp. 747-752, 2012.

[11] Y. Hu and P. Georgiou, "A robust isfet ph-measuring front-end for chemical reaction monitoring," IEEE Transactions on Biomedical Circuits and Systems, vol. 8, no. 2, pp. 177-185, 2014.
[12] Y. Liu, A. Al-Ahdal, P. Georgiou, and C. Toumazou, "Minimal readout scheme for isfet sensing arrays based on pulse width modulation," Electronics letters, vol. 48, no. 10, pp. 548-549, 2012.

[13] P. Bergveld, "Thirty years of isfetology* $1::$ What happened in the past 30 years and what may happen in the next 30 years," Sensors and Actuators B: Chemical, vol. 88, no. 1, pp. 1-20, 2003.

[14] Y. Liu, T. G. Constandinou, and P. Georgiou, "A $32 \times 32$ isfet array with in-pixel digitisation and column-wise tdc for ultra-fast chemical sensing," in 2019 IEEE International Symposium on Circuits and Systems (ISCAS). IEEE, 2019, pp. 1-5.

[15] K. B. Parizi, X. Xu, A. Pal, X. Hu, and H. P. Wong, "Isfet ph sensitivity: Counter-ions play a key role," Scientific reports, vol. 7, p. 41305, 2017.

[16] N. Moser, T. S. Lande, C. Toumazou, and P. Georgiou, "Isfets in cmos and emergent trends in instrumentation: A review," IEEE Sensors Journal, vol. 16, no. 17, pp. 6496-6514, 2016.

[17] J. Zeng and P. Georgiou, "Current-mode isfet array with row-parallel adcs for ultra-high speed ion imaging," in 2019 IEEE International Symposium on Circuits and Systems (ISCAS). IEEE, 2019, pp. 1-5.

[18] Y. Liu and C. Toumazou, "An isfet based sensing array with sensor offset compensation and ph sensitivity enhancement," in Proceedings of 2010 IEEE International Symposium on Circuits and Systems. IEEE, 2010, pp. 2283-2286.

[19] P. Georgiou and C. Toumazou, "Isfet threshold voltage programming in cmos using hot-electron injection," Electronics letters, vol. 45, no. 22, pp. 1112-1113, 2009.

[20] M. J. Milgrew and D. R. Cumming, "Matching the transconductance characteristics of cmos isfet arrays by removing trapped charge," IEEE Transactions on Electron Devices, vol. 55, no. 4, pp. 1074-1079, 2008.

[21] A. Al-Ahdal and C. Toumazou, "Isfet threshold voltage programming in cmos using electron tunnelling," Electronics letters, vol. 47, no. 25, pp. 1398-1399, 2011.

[22] R. Van Hal, J. C. Eijkel, and P. Bergveld, "A general model to describe the electrostatic potential at electrolyte oxide interfaces," Advances in colloid and interface science, vol. 69, no. 1-3, pp. 31-62, 1996.

[23] B. A. Minch, "Construction and transformation of multiple-input translinear element networks," IEEE Transactions on Circuits and Systems I: Fundamental Theory and Applications, vol. 50, no. 12, pp. 1530 1537, 2003.

[24] J. Ramirez-Angulo, G. Gonzalez-Altamirano, and S. Choi, "Modeling multiple-input floating-gate transistors for analog signal processing," in Proceedings of 1997 IEEE International Symposium on Circuits and Systems. Circuits and Systems in the Information Age ISCAS'97, vol. 3. IEEE, 1997, pp. 2020-2023.

[25] C. Jakobson, M. Feinsod, and Y. Nemirovsky, "Low frequency noise and drift in ion sensitive field effect transistors," Sensors and Actuators B: Chemical, vol. 68 , no. $1-3$, pp. $134-139,2000$

[26] L. Bisdounis, S. Nikolaidis, and O. Koufopavlou, "Analytical transient response and propagation delay evaluation of the cmos inverter for shortchannel devices," IEEE Journal of Solid-State Circuits, vol. 33, no. 2, pp. 302-306, 1998.

[27] B. Razavi, Fundamentals of microelectronics. Wiley, 2008

[28] L. B. Leene and T. G. Constandinou, "A $0.006 \mathrm{~mm} 21.2 \backslash \mu \mathrm{w}$ analogto-time converter for asynchronous bio-sensors," IEEE Journal of SolidState Circuits, vol. 53, no. 9, pp. 2604-2613, 2018.

[29] R. R. Harrison and C. Charles, "A low-power low-noise cmos amplifier for neural recording applications," IEEE Journal of solid-state circuits, vol. 38, no. 6, pp. 958-965, 2003.

[30] J. M. Rabaey, A. P. Chandrakasan, and B. Nikolic, Digital integrated circuits. Prentice hall Englewood Cliffs, 2002, vol. 2.

[31] K. Wang, Y. Liu, C. Toumazou, and P. Georgiou, "A tdc based isfet readout for large-scale chemical sensing systems," in Biomedical Circuits and Systems Conference (BioCAS), 2012 IEEE. IEEE, 2012, pp. 176-179. 


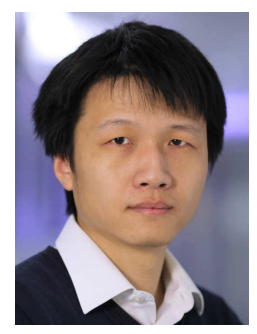

Yan Liu (AM'08-M'12) received the B.Eng. degree in 2006 from Zhejiang University, China, the M.Sc. degree in 2007 and Ph.D. in 2012 from Electrical and Electronic Engineering at Imperial College London, UK. From 2012 to 2019, he was research associate then research fellow in Centre of Bioinspired technology, Electrical and Electronic Engineering at Imperial College London. From 2019, he is also with Department of Micro-Nano Electronics, Shanghai Jiaotong University, China. His research area includes: CMOS based lab-on-chip devices and platforms, brain machine interface, and novel mixed signal circuits for biomedical applications.

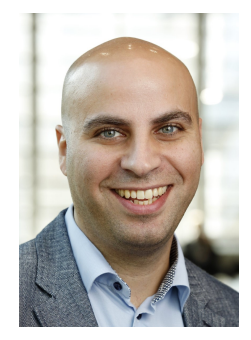

Timothy G. Constandinou (AM'98-M'01-SM'10) received the B.Eng. and Ph.D. degrees in electronic engineering from Imperial College London, in 2001 and 2005 , respectively. He is currently a Reader of Neural Microsystems within the Circuits and Systems Group, Department of Electrical and Electronic Engineering at Imperial College London and also the Deputy Director of the Centre for Bio-Inspired Technology. His current research interests include neural microsystems, neural prosthetics, brain machine interfaces, implantable devices, and low-power microelectronics. He is a fellow of the IET, a chartered engineer, and member of the IoP. Within the IEEE, he serves on several committees/panels, regularly contributing to conference organization, technical activities, and governance. He chairs the IEEE Sensory Systems Technical Committee, is a member of the IEEE BioCAS Technical Committee, IEEE Brain Initiative Steering Committee, and serves on the IEEE Circuits and Systems Society Board of Governors for the term 2017-2019. He was the technical program Co-Chair of the 2010, 2011 and 2018 IEEE BioCAS conferences, General Chair of the BrainCAS 2016 and NeuroCAS 2018 workshops, Special Session Co-Chair of the 2017 IEEE ISCAS Conference, and Demonstrations Co-Chair of the 2017 BioCAS Conference. He is currently an associate editor of the IEEE Transactions on Biomedical Circuits and Systems.

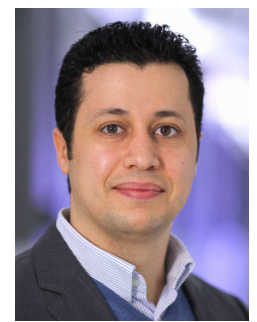

Pantelis Georgiou (A'05-M'08-SM'13) received the M.Eng. degree in electrical and electronic engineering and the Ph.D. degree from Imperial College London (ICL), London, U.K., in 2004 and 2008, respectively. He is currently a Reader with the Department of Electrical and Electronic Engineering, ICL, where he is also the Head of the Bio-Inspired Metabolic Technology Laboratory, Centre for BioInspired Technology. He has made significant contributions to integrated chemical-sensing systems in CMOS, conducting pioneering work on the development of ISFET sensors, which has enabled applications, such as point-of-care diagnostics and semiconductor genetic sequencing and has also developed the first bio-inspired artificial pancreas for treatment of Type I diabetes using the silicon-beta cell. His research includes bio-inspired circuits and systems, CMOS-based Lab-on-Chip technologies, and application of microelectronic technology to create novel medical devices. He received the IET Mike Sergeant Medal of Outstanding Contribution to Engineering in 2013. In 2017, he received the IEEE Sensors Council Technical Achievement Award. He is a member of the IET. He serves on the BioCAS and Sensory Systems Technical Committees for the IEEE CAS Society. He is also the CAS representative on the IEEE Sensors Council and the IEEE Distinguished Lecturer in circuits and systems. 